\title{
Value of CD44 immunostaining (IHC) as a surrogate in the differentiation of MYC-positive Burkitt lymphoma (BL) and Burkitt lymphoma-like (BLL) from MYC-negative Diffuse Large B-Cell Lymphoma (DLBCL) in a resource constrained clinical setting LW Ayers*1, M Ogwang ${ }^{2}$, W Zhou ${ }^{1}$, SM Mbulaiteye ${ }^{3}$ and K Bhatia ${ }^{3}$
}

Address: ${ }^{1}$ The Ohio State University, Columbus, Ohio, USA, ${ }^{2}$ St. Mary's Hospital Lacor, Gulu, Uganda and ${ }^{3}$ National Cancer Institute, Bethesda, Maryland, USA

* Corresponding author from I I th International Conference on Malignancies in AIDS and Other Acquired Immunodeficiencies (ICMAOI): Basic, Epidemiologic, and Clinical
Research

Bethesda, MD, USA. 6-7 October 2008

Published: 17 June 2009

Infectious Agents and Cancer 2009, 4(Suppl 2):P6 doi:I0.1 186/1750-9378-4-S2-P6

This abstract is available from: http://www.infectagentscancer.com/content/4/S2/P6

(c) 2009 Ayers et al; licensee BioMed Central Ltd.

\section{Background}

Both BL and DLBCL are prevalent in HIV/AIDS patients and the correct classification of these two subtypes of Non-Hodgkin's lymphomas (NHL) particularly between DLCBL with high proliferation and BL, is critical to chemotherapy choice. Transcriptional gene expression profiling of sporadic and endemic BL by Stein et al (Berlin) identified a molecular signature for BL that included a down regulation of CD44 expression in a group of lymphomas from Africa. Immunophenotyping of formalin-fixed paraffin-embedded (FFPE) BL tissues by this group demonstrated difficulties in correctly classifying BL using morphology, BCL-2 (immunohistochemistry - IHC) presence and MYC (fluorescent in situ hybridization - FISH) absence. The differential expression of $\mathrm{CD} 44$, albeit nonmembranous, suggests an intricate regulatory circuit that allows for low expression, supporting the possibility that CD44 plays a role in BL/BLL dissemination. CD44 (IHC) could be a valuable marker to aid classification of tumors whose morphologic and phenotypic overlap is among $\mathrm{BL}$, BLL and DLBCL. CD44 absence clearly separates MYC+ BL/BLL and MYC-DLBCL. DLBCL, Hodgkin's lymphoma (HL) and normal lymphocytes from lymphoid mantle/ marginal cell and interfollicular T-cell-rich zones should be strongly CD44 positive.
Materials and methods

We evaluated 128 FFPE lymphoma tissue biopsy collected at St. Mary's Lacor Hospital, Gulu, Uganda, Africa, from 1994 to 2007 from tissue microarray (TMA) blocks constructed on site using the UNITMA Quick-Ray System (Seoul, South Korea). The completed TMA blocks were sectioned and stained for H\&E, IHC including: CD44, CD43，CD45, CD45RO, MUM-1，CD138，TdT, CD5, CD3, CD20, CD79a, CD10, BCL-2, BCL-6, KI67 (MIM-1), HHV-8 (LANA-1), IgM, CD30, CD15, CD68, CD23, Vimentin, CD34, EMA, CD34, Cyclin D1, BIM and p53; in situ hybridization (ISH) for EBV (EBER) and kappa and lambda light chains and FISH break apart assay for CMYC translocation.

\section{Results}

Of the 128 core samples collected (one per case), 107 could be interpreted. Ninety-two had results for CD44; 15 could not be read for $\mathrm{CD} 44$ due to missing tissue or necrosis. Seventy-one $(66.4 \%)$ were negative for significant CD44 staining of tumor cells. Included were: $53 \mathrm{BL}, 10$ Burkitt lymphoma-like (BLL) and eight other (two small cell lymphomas, one marginal cell lymphoma, one lymphoblastic lymphoma, one follicular lymphoma, two normal tissues and one alveolar rhabdomyosarcoma). Twenty-one (19.6\%) core samples were CD44 positive. Included were four DLBCL and 17 other (two small cell 
lymphomas, one gut associated lymphoma, one anaplastic large cell lymphoma, one histocyte rich lymphoma, one peripheral $\mathrm{T}$ cell lymphoma, three lymph nodes, three HD, two follicular lymphomas, and three normal tissues). Of note, supporting tissue in some BLL samples were positive for CD44 unlike the BL group, but the BLL tumor cells did not show membrane staining as seen with the DLBCL cells. C-MYC (FISH) probe produced no signals in the 42 biopsies collected prior to 2003. The $27 \mathrm{BL} / \mathrm{BLL}$ samples from 2003 to 2007 were C-MYC positive (17 or $73.9 \%$ ) or C-MYC negative (six or $26.1 \%$ ) except for four instances where the tissue core section was not present on the test slide. DLBCL samples were C-MYC negative. Most BL core sections $(89.6 \%)$ and BLL core sections (90\%) were EBV positive while all DLBCL core sections were EBV negative. The $\mathrm{BL} / \mathrm{BLL}$ core sections were BCL-2 negative and usually BCL-6 positive (87.5\%).

\section{Conclusion}

CD44 absence on BL/BLL cells aligned C-MYC translocation, EBV-positive tumor cells and absence of BCL-2 with the BL group. The DLBCL group was strongly positive for CD44 cell membrane staining and variably positive for BCL-2, MUM-1 and CD10. Where BL/BLL presented as infiltrating cells into various supporting tissues rather than as monomorphic tumor, CD44 staining was insufficient to distinguish the BL/BLL cells but could be used along with other tests such as CD20 and BCL-6 to characterize this subtype. Our results suggest that CD44 is of value when used along with tumor cell morphology, as a clarifying additional test or as a surrogate for C-MYC testing in resource constrained settings to assure accurate separation of BL/BLL from DLBCL.

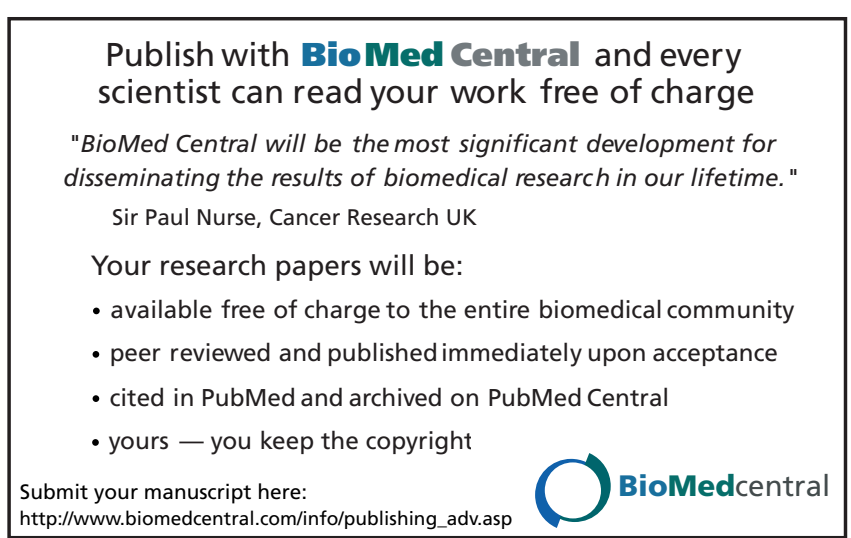

Page 2 of 2

(page number not for citation purposes) 